\title{
GROUND-BASED MEASUREMENTS AND VALIDATION PROTOCOLS FOR FLEX
}

\author{
Elizabeth M. Middleton ${ }^{1+}$, K. Fred Huemmrich ${ }^{2}$, Petya K.E. Campbell ${ }^{2}$, Qingyuan Zhang ${ }^{3}$, \\ David R. Landis ${ }^{4}$, Cris Garrish ${ }^{2}$, Lawrence Ong ${ }^{5}$, and Craig Daughtry ${ }^{6}$ \\ ${ }^{1}$ NASA/Goddard Space Flight Center, Greenbelt, MD 20771, USA \\ ${ }^{2}$ University of Maryland Baltimore County, Baltimore, MD 21228, USA \\ ${ }^{3}$ Universities Space Research Association, Columbia, MD 21046, USA \\ ${ }^{4}$ Global Science \& Technology, Greenbelt, MD 20770, USA \\ ${ }^{5}$ Science Systems and Applications, Inc., Lanham, MD 20706, USA \\ ${ }^{6}$ USDA-ARS Hydrology and Remote Sensing Laboratory, Beltsville, MD 20705, USA \\ † Corresponding author, e-mail: Elizabeth.M.Middleton@nasa.gov
}

\begin{abstract}
The upcoming ESA Fluorescence Explorer (FLEX) mission will incorporate ground-based validations for fluorescence parameters and reflectance indices, drawing on an international network of sensors located at eddy covariance tower sites. A program has been initiated by the OPTIMISE program to develop methods and protocols for this network. A sensor system suite under evaluation by OPTIMISE includes the FLoX hyperspectral spectroradiometers. The NASA team at GSFC is participating in this experiment and we report first results from the 2017 summer measurements made above the canopy at the USDA/ARS Beltsville cornfield using the DFLoX and two other leaf-level measurement systems, the MONI-PAM and the FluoWat.
\end{abstract}

Index terms: FLEX, fluorescence, SIF, FluoWat, MONIPAM, DFLoX

\section{INTRODUCTION}

In recent years, several coarse spatial resolution satellites designed to monitor atmospheric constituents globally have been used to retrieve solar-induced chlorophyll fluorescence (SIF) in the near-infrared spectral region. It has proved difficult to validate these $\leq 0.5$ degree observations using point source surface data such as Gross Primary Production (GPP) measurements available from eddy covariance networks. In late 2015, the European Space Agency (ESA) selected the FLuorescence EXplorer (FLEX) as its Explorer 8 mission, the first orbital mission specifically designed to obtain chlorophyll fluorescence [1], with an anticipated launch in $2022 / 2023$. The FLEX mission will be a tandem mission between the operational ESA/Sentinel-3C (S-3C) satellite and the FLORIS free flyer for SIF, which together will provide the full chlorophyll fluorescence emission spectrum $(650-800 \mathrm{~nm})$ at the ecologically relevant scale of $300 \mathrm{~m}$. The FLORIS' two imaging spectrometers will measure radiance at high spectral resolution $(\sim 0.3 \mathrm{~nm})$ with its Narrow Band sensor in the regions of the two atmospheric oxygen absorption bands centered at $687\left(\mathrm{O}_{2}-\mathrm{B}\right.$, red SIF $)$ and 760 $\mathrm{nm}\left(\mathrm{O}_{2}-\mathrm{A}\right.$, far-red SIF), and surface reflectance in the visi- ble through near-infrared region at $\sim 3-5 \mathrm{~nm}$ resolution with its Wide Band sensor. Supporting measurements will be provided by the S-3C instruments. A program to support ground-based data validations for the FLEX mission is currently under development by the OPTIMISE ${ }^{1}$ international program. This initiative is deploying instruments capable of monitoring chlorophyll fluorescence and related spectral responses at selected eddy covariance/meteorology tower and other intensive study sites around the world. The first such sensor system suite sponsored by ESA and OPTIMISE is now in use at $\sim 15$ sites, including our long-term maize study site in the USA $[2-5,9]$. The suite includes the FLuorescence bOX (FLoX) and Dual FLoX (DFLoX), JB Hyperspectral Devices, Inc., Düsseldorf, Germany [6]. NASA/GSFC deployed its DFLoX during the 2017 growing season in a rain-fed cornfield, with supporting measurements, at the USDA Agriculture Research Center in Beltsville, MD. We provide the first analyses of those datasets.

\section{METHODS}

The cornfield (Zea mays L.) was planted in early June and fertilized at best management practices rate $(100 \% \mathrm{~N})$ in mid-June at the USDA-ARS Beltsville site. The young crop was $\sim 0.5 \mathrm{~m}$ high at the start of our measurements in early July when the NASA/GSFC team established a DFLoX system on an elevated support structure in a fixed location. The system acquired continuous daytime measurements for 81 days at one meter above the canopy (adjusted as needed) from July 10 until October 12, with a 12 day gap in late July/early August when the DFLoX was deployed elsewhere to support an airborne campaign. In a nearby section of the field, a Monitoring Pulse Amplified Modulator (MONI-PAM) system (Heinz Walz, GmbH, Effeltrich, Germany) was established to measure fluorescence on 5 individual upper canopy leaves, sampling at 10 minute intervals throughout the day. Leaf-level SIF was determined using a custom FluoWat leaf clip [7] paired with an ASD spectroradiometer (Analytical Spectral Devices, Longmont, CO) for upper layer leaves. Here, we report results from leaves in the $\mathrm{N}$

${ }^{1}$ ESSEM COST Action ES1309 (http://optimise.dcs.aber.ac.uk/) 
plots, examining the $150 \%$ versus $0 \% \mathrm{~N}$ (i.e., over-fertilized vs. under-fertilized) treatments. In both the main field and the $\mathrm{N}$ plots, supporting leaf level data and canopy characteristics were acquired (e.g., leaf morphology, chlorophyll content, optical properties, gas exchange, pulse activated fluorescence, plant height, etc.). An USDA eddy covariance tower was located in the main field. We provide an initial analysis of the DFLoX canopy observations, and leaf-level data from the MONI-PAM and the FluoWat, acquired during this first USA validation experiment, in support of developing FLEX protocols.

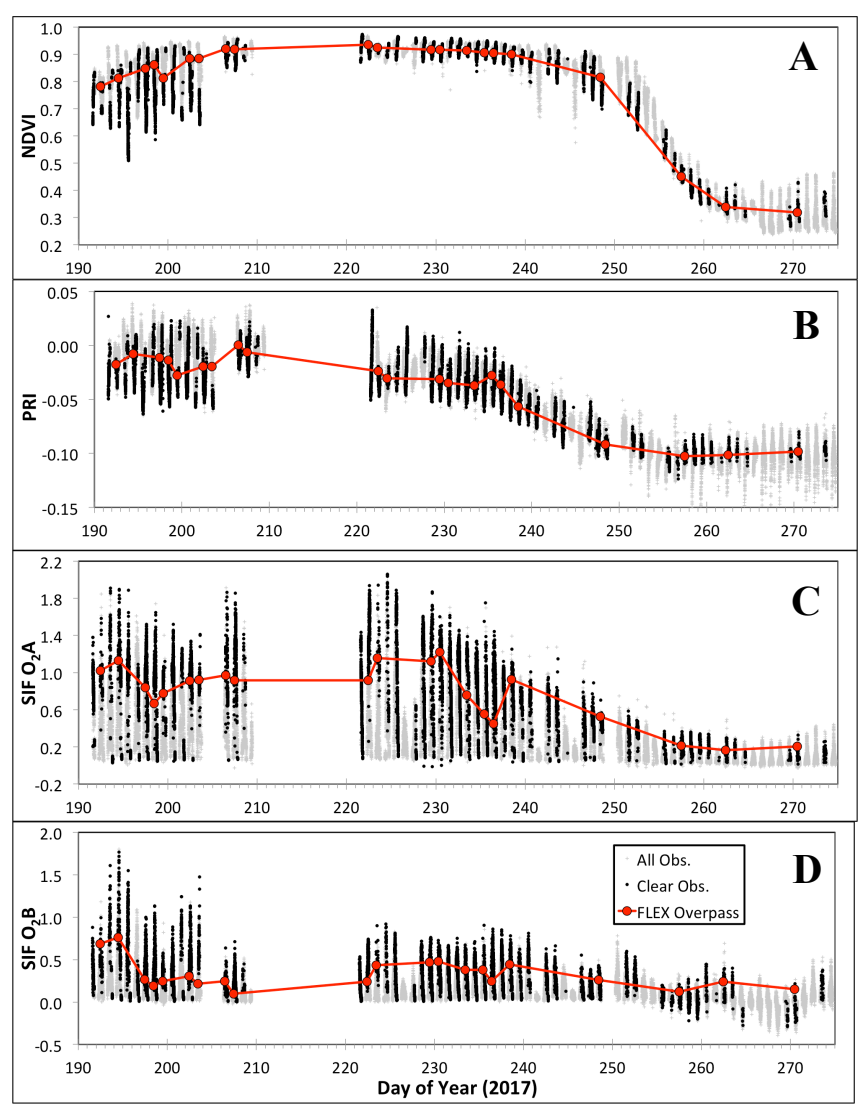

Fig. 1. DFLoX data from the USDA/Beltsville cornfield (100\% N) for the 2017 growing season: [A] NDVI; [B] PRI; [C] far-red $\mathrm{SIF}\left(\mathrm{O}_{2}-\mathrm{A}\right)$; [D] red $\mathrm{SIF}\left(\mathrm{O}_{2}-\mathrm{B}\right)$. Gray points are all observations, black points are observations collected under clear conditions, red points are clear observations collected near the 11 AM FLEX overpass time. SIF $\left(\mathrm{mW} / \mathrm{m}^{2} / \mathrm{sr} / \mathrm{nm}\right)$ was calculated using the iFLD method [8].

From the DFLoX data (Fig. 1), we determined the canopy spectral trends throughout each measurement day across the growing season for four spectral parameters: the Normalized Difference Vegetation Index (NDVI); the Photochemical Reflectance Index (PRI); and the SIF in the $\mathrm{O}_{2}$-A and the $\mathrm{O}_{2}$ - $\mathrm{B}$ bands. The diurnal and seasonal variation of these four canopy variables were examined. We also tracked the seasonal trends for values corresponding to those acquired at the expected FLEX local overpass time of $\sim 11 \mathrm{AM}$, and simultaneous PRI and SIF responses (Fig. 2). The leaf-level fluo- rescence responses acquired with the MONI-PAM system were also examined over the season (Fig. 3). The leaf-level SIF emission spectra acquired with the FluoWat (Fig. 4) were processed according to published methods [7].
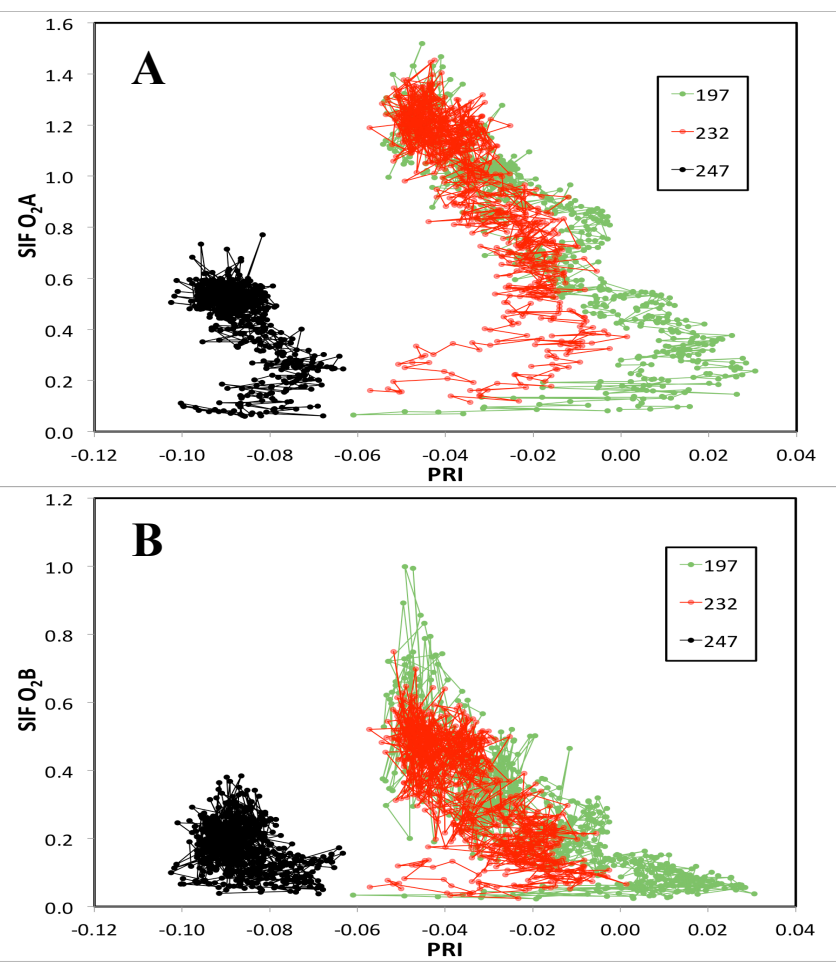

Fig. 2. The DFLoX observations for $\operatorname{SIF}\left(\mathrm{mW} / \mathrm{m}^{2} / \mathrm{sr} / \mathrm{nm}\right)$ vs. PRI are plotted for three clear days from different growth stages: DOY 197 (July 16, green points/line), DOY 232 (Aug 20, red points/line), and DOY 247 (Sep 4, black points/line): [A] far-red $\operatorname{SIF}\left(\mathrm{O}_{2}-A\right)$; and [B] red $\operatorname{SIF}\left(\mathrm{O}_{2}-\mathrm{B}\right)$.

\section{RESULTS AND DISCUSSION}

The DFLoX acquired hundreds of observations daily. We describe results here related to the 11 AM EST observations. As a baseline, when the canopy was fully developed by midJuly (DOY 208) the NDVI had attained a relatively high and stable value of $0.93 \pm 0.02$ which was sustained for over a month until DOY 240, after which it declined steadily until DOY 267 when a second stable value of $\sim 0.35 \pm 1.0$ was reached (Fig. 1A). The highest average daily PRI value $(0.0 \pm$ 0.02 ), expressing low physiological stress, also occurred on DOY 208, after which a slow decline occurred as stress increased until DOY 252 when a stable value of $-0.08 \pm 0.03$ was reached during senescence (Fig. 1B). SIF was highest for the young canopy and for the reproductive growth stage for both the far-red SIF $\left(\sim 1.3 \mathrm{~mW} / \mathrm{m}^{2} / \mathrm{sr} / \mathrm{nm}\right)$ and red SIF $(\sim 0.5$ $\mathrm{mW} / \mathrm{m}^{2} / \mathrm{sr} / \mathrm{nm}$ ) (Fig. 1C, D). Red SIF was lowest $(0.2$ $\mathrm{mW} / \mathrm{m}^{2} / \mathrm{sr} / \mathrm{nm}$ ) during the fully developed vegetated stage (DOY 194-200+). SIF dropped during two episodes (DOY $\sim 198,236$ ). We show that the PRI and SIF (Fig. 2A, B) changed together during 3 clear sky days: those in early and mid-season were distinctly different from the late season day. 

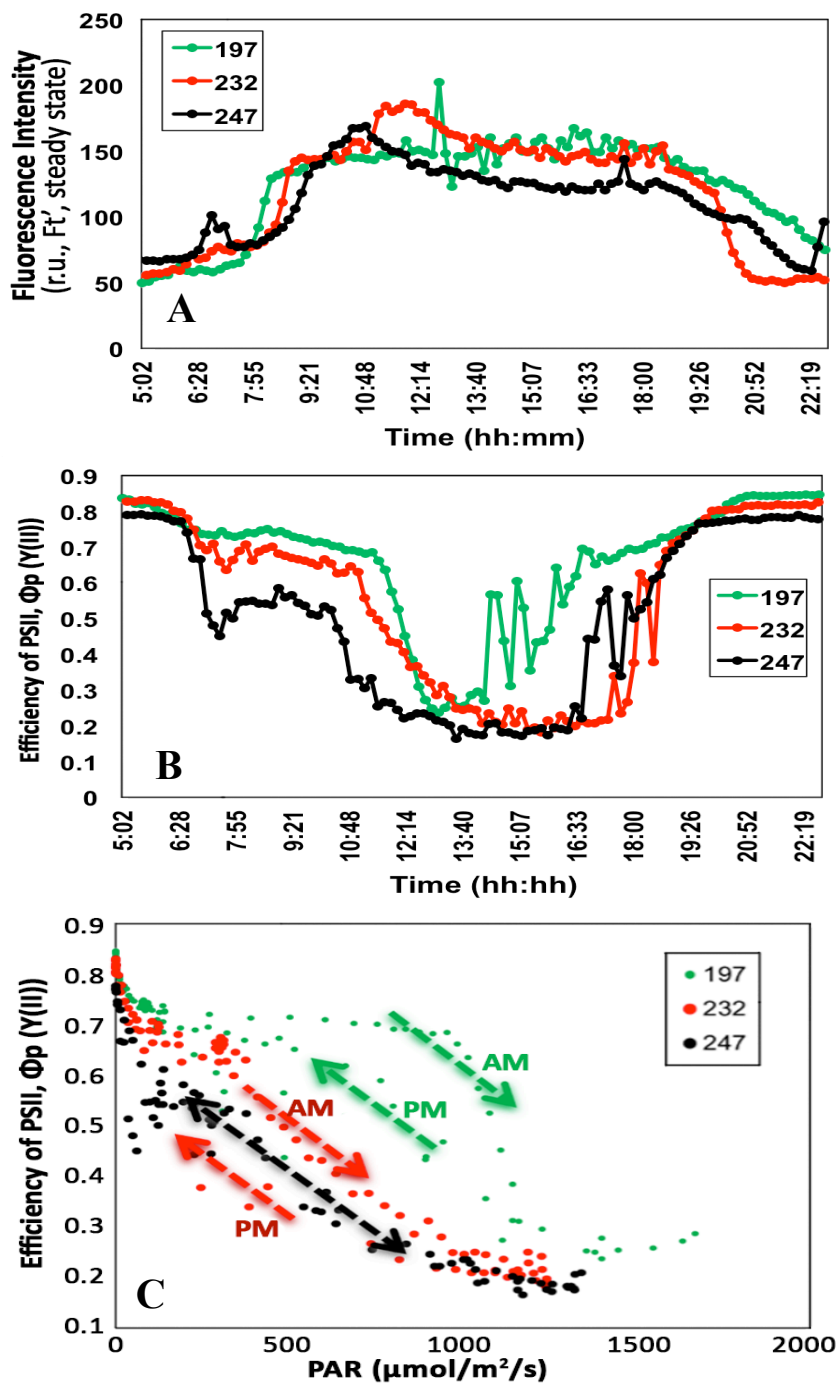

Fig. 3. MONI-PAM measurements on fully sunlit leaves: [A] diurnal and seasonal differences in steady state chlorophyll fluorescence intensity $\left(F t^{\prime}\right) ;[\boldsymbol{B}]$ diurnal and seasonal differences in photochemical yield, $\Phi p(Y(I I)) ;[\boldsymbol{C}]$ diurnal and seasonal changes in $\Phi p$ as a function of PAR.

The automated MONI-PAM system acquired more than 10,000 measurements under varying weather and illumination conditions. Here, we examine the observations obtained on fully sunlit leaves (Fig. 3) on the same clear days for which the DFLoX measurements are presented above. The short term variations in steady state chlorophyll fluorescence emissions (Ft') (Fig. 3A) and the photosynthetic efficiency of Photosystem II, PSII (Фp)(Fig. 3B) depict similar daily trends on each of these three days, representing different growth stages. Daily $\mathrm{Ft}^{\prime}$ (Fig. 3A) was relatively stable during mid-days. While predawn Ft' was approximately the same on all three days (50-75 r.u.), the mid-day maximum $\mathrm{Ft}^{\prime}$ values on the early season day (DOY 197) occurred almost three hours earlier ( $~ 8$ AM) than on the mid-season day (DOY 232) when maximum $\mathrm{Ft}^{\prime}$ was delayed until noon, and reverted to an earlier time by the end of the growing season (DOY 247, 10 AM).
Photosynthetic quantum efficiencies for PSII ( $\Phi$ p, Fig. 3B) varied and were bowl-shaped diurnally. Morning responses were high early in the season (DOY 197), declined to mid-season, and then further for DOY 247. On DOY 197, $\Phi p$ recovered from the mid-day depression 2-3 hours earlier than on the other two days (Fig. 3B), but all had similarly high $\Phi p$ at day's end. The most interesting trends for PSII efficiency were observed as a declining function of incoming PAR intensity (Fig. 3C), with hysteresis occurring for morning vs. afternoon responses on all three days. Morning efficiencies declined to attain daily minima near noon, before increasing throughout the afternoons to regain original values. Morning (declining) values were higher than afternoon (increasing) values for DOY 197 and 232, but equal for morning/afternoon during senescence. Consequently, the MONI-PAM collection captured the metabolic changes in the PSII operating efficiency which varied diurnally and seasonally due to changes in light regimes, temperature, and foliar photosynthetic pigment content.
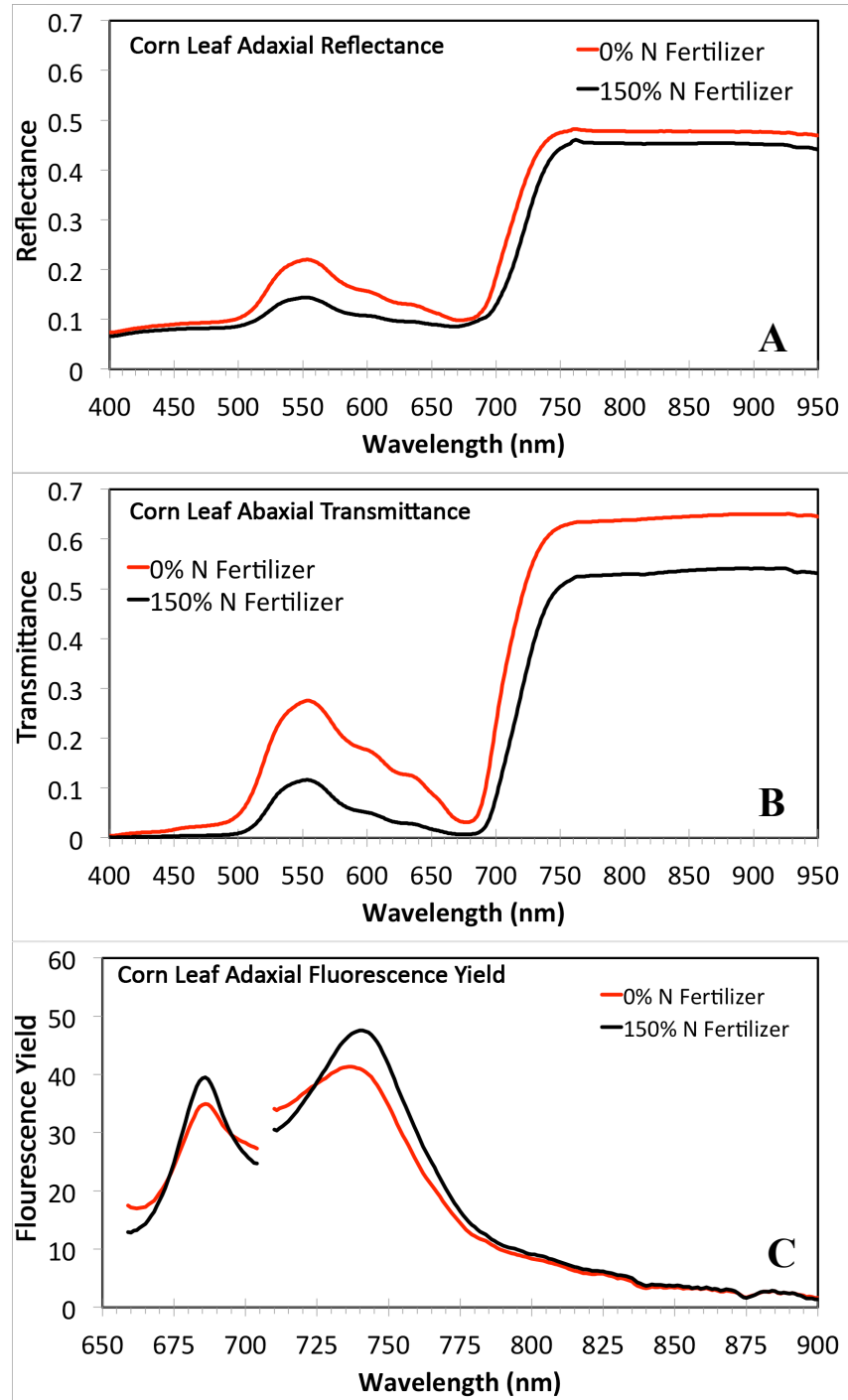


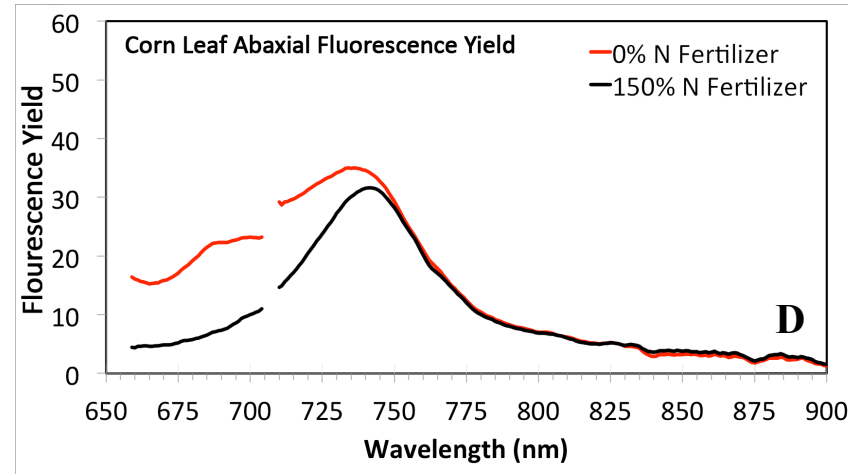

Fig. 4. In situ corn leaf spectra collected using FluoWat leaf clip for: [A] leaf adaxial reflectance; [B] leaf abaxial transmittance; [C]; leaf adaxial fluorescence yield; and [D] leaf abaxial fluorescence yield. Spectra in $\boldsymbol{C}$ and $\boldsymbol{D}$ were computed as $(F / A P A R) * 10^{6}$. The data were collected on 8/25/15 between 10-12 AM, as an average of three leaves.

The FluoWat measurements revealed higher leaf reflectance and transmittance in the nitrogen-deficient $(0 \% \mathrm{~N})$ plants (Fig. 4A, B) and for fluorescence yield (F/APAR) determined at the leaf undersides for wavelengths $<750 \mathrm{~nm}$ (Fig. 4D). Upper surfaces had higher fluorescence yields for the $150 \%$ group at both red and far-red peaks (Fig. 4C).

\section{CONCLUSIONS}

Our results demonstrate the value of continuous groundbased measurements at an intensely studied research site. In particular, this type of measurement suite for canopy and leaf level measurements is essential for interpretation of future FLEX measurements [5, 9-10], and especially for Level 2 and higher products. Our site also has a 10 meter tower instrumented with spectrometers to capture directional fluorescence and reflectance measurements, referred to as the GSFC FUSION system. We reported last year [11] that a correction is necessary for the oxygen concentration in the atmospheric column when the distance between the vegetation and the sensor and the reference is $\geq 10 \mathrm{~m}$. We plan to integrate the FUSION data with the eddy covariance data, and the three dataset types described in this report. Our goal is to develop a useful and practical ground validation system in support of FLEX and other vegetation missions.

\section{REFERENCES}

[1] Drusch, M., J. Moreno, U. del Bello, R. Franco, Y. Goulas, A. Huth, S. Kraft, E. Middleton, F. Miglietta, G. Mohammad, L. Nedbal, U. Rascher, D. Schuttemeyer, and W. Verhoef (2016). The Fluorescence EXplorer (FLEX) Mission Concept- ESA's Earth Explorer 8 (EE8), IEEE Tran. Geo. Rem. Sensing (TGARS), 55(3): 1273-1284, [doi:10.1109/TGRS.2016.2621820].

[2] Middleton, E.M., L.A. Corp, and P.K.E. Campbell (2008). Comparison of measurements and FluorMOD simulations for solar induced chlorophyll fluorescence and reflec- tance of a corn crop under nitrogen treatments, Int'l J. Rem. Sensing, Special Issue for $2^{\text {nd }}$ Recent Advances in Quantitative Remote Sensing (RAQRSII), 29(17): 5, 193-213.

[3] Corp, L.A., J.E. McMurtrey, E.M. Middleton, E.W. Chappelle, and C.S.T. Daughtry (2003). Fluorescence sensing systems: in vivo detection of biophysical variations in field corn due to nitrogen supply, Rem. Sens. Environ., 86: 470-479.

[4] Cheng, Y.-B., E.M. Middleton, Q. Zhang, K.F. Huemmrich, P.K. Campbell, L.A. Corp, B.D. Cook, W.P. Kustas, and C.S. Daughtry (2013). Integrating solar induced fluorescence and the photochemical reflectance index for estimating gross primary production in a cornfield. Rem. Sensing, 5(12), 6857-6879.

[5] Middleton, E.M., Y.-B. Cheng, P.E. Campbell, K.F. Huemmrich, L.A. Corp, S. Bernardes, Q. Zhang, D.R. Landis, W.P. Kustas, C.S.T. Daughtry, and A.L. Russ (2016). Multi-angle hyperspectral observations with SIF and PRI to detect plant stress \& GPP in a cornfield. Proceedings, $\boldsymbol{9}^{\text {th }}$ EARSeL SIG Workshop on Imaging Spectroscopy, Luxembourg City, Luxembourg, April 2015, 11 pp., 2/16.

[6] Julitta, T., et al. 2017. Accurate measurements of fluorescence in the $\mathrm{O}_{2}$-A and $\mathrm{O}_{2}$-B bands using the FloX spectroscopy system - results and prospects. Poster, Green House Gases Flux Workshop, Potsdam, Germany, 24-26 October 2017.

[7] Van Wittenberghe, S., L. Alonso, J. Verrelst, J. Moreno, and R. Samson (2015). FluoWat bidirectional sun-induced chlorophyll fluorescence emission is influenced by leaf structure and light scattering properties: A bottom-up approach. Rem. Sensing of Environ. 158: 169-179.

[8] Alonso, L., et al. (2008). Improved Fraunhofer line discrimination method for vegetation fluorescence quantification, Geosci. Rem. Sening Letters, IEEE, 5 (4), 620-624.

[9] Campbell, P.K.E., E.M. Middleton, L.A. Corp, C. van der Tol, K.F. Huemmrich, M.P. Cendrero-Mateo and J. Leifeld (2014). Diurnal and phenological changes in vegetation fluorescence and reflectance, indicative of vegetation photosynthetic properties and function. In, Proceedings of the 5th International Workshop on Remote Sensing of Vegetation Fluorescence, Paris, France, April 2014, 12 pp.

[10] van der Tol, C., J.A. Berry, P.K.E. Campbell, and U. Rascher (2015). Models of fluorescence and photosynthesis for interpreting measurements of solar-induced chlorophyll fluorescence: open access. J. Geophys. Research: Biogeosciences, 119(12), 2312-2327; doi: 10.1002/2014JG002713.

[11] Sabater N., E.M. Middleton, Z. Malenovsky, L. Alonso, J. Verrelst, K.F Huemmrich, P.K.E. Campbell, W.P. Kustas, J. Vicent, S. Van Wittenberghe, and J. Moreno (2017). Oxygen transmittance correction for solar-induced chlorophyll fluorescence measured on proximal sensing: application to the NASA-GSFC Fusion tower. 4 pp. IGARSS 2017, Ft. Worth, TX. 